\title{
Brain activation during processing of angry facial expressions in patients with alcohol dependency
}

\author{
Mi-Sook Park', Sook-Hee Kim², Sunju Sohn ${ }^{3}$, Gap-Jung Kim ${ }^{4}$, Yeon-Kyu Kim ${ }^{{ }^{*}}$ and Jin-Hun Sohn ${ }^{*}$
}

\begin{abstract}
Background: Alcoholism is associated with abnormal anger processing. The purpose of this study was to investigate brain regions involved in the evaluation of angry facial expressions in patients with alcohol dependency.

Methods: Brain blood-oxygenation-level-dependent (BOLD) responses to angry faces were measured and compared between patients with alcohol dependency and controls.

Results: During intensity ratings of angry faces, significant differences in BOLD were observed between patients with alcohol dependency and controls. That is, patients who were alcohol-dependent showed significantly greater activation in several brain regions, including the dorsal anterior cingulate cortex (dACC) and medial prefrontal cortex (MPFC).

Conclusions: Following exposure to angry faces, abnormalities in AACC and MPFC activation in patients with alcohol dependency indicated possible inefficiencies or hypersensitivities in social cognitive processing.
\end{abstract}

Keywords: Alcoholism, Anger, BOLD, Anterior cingulate cortex, Medial prefrontal cortex

\section{Background}

Alcohol serves many purposes for both social drinkers and individuals with alcohol-abuse disorders. As specified in various alcohol-use scales, these purposes include increasing sociability, overcoming shyness or uneasiness, "joining the group," forgetting about problems, getting drunk or intoxicated, or simply enjoying the taste. However, for many individuals suffering from alcoholism, the urge to drink can be associated with heightened anger. While some turn to alcohol in an attempt to reduce feelings of anger, others feel justified to drink because someone has made them feel angry. These statements are supported by several studies using valid scales. For example, as measured by several State-Trait Anger Expression Inventory subscales [1,2] and the Profile of Mood States scale [3], individuals who abuse alcohol often present higher levels of anger than "normal" individuals.

Parrott et al. [4] found that individuals who drink alcohol and have a tendency toward chronic anger are

\footnotetext{
* Correspondence: yk-kim@design.kyushu-u.ac.jp; jhsohn@cnu.ac.kr ${ }^{5}$ Department of Industrial Design, Graduate school of Integrated Frontier Science, Faculty of Design, Kyushu University, 4-9-1 Shiobaru, Minami-ku, Fukuoka 815-8540, Japan

'Department of Psychology, Chungnam National University, 99 Daehak-ro, Yuseong-gu, Daejeon 305-764, South Korea

Full list of author information is available at the end of the article
}

much more likely to become enraged when drinking alcohol. More recently, Kelly et al. [5] followed the progress of more than 1,700 patients with alcohol dependence who were being treated in clinical trials every 3 months for over a year and found that they were more likely to become frustrated and angry compared to individuals without alcohol-abuse disorders. This leaves researchers to question "why" anger plays a critical role in the lives of many individuals with alcoholism and "how" they are different from normal people in processing anger. One explanation is that people with alcoholism may have trouble appropriately expressing their emotion, as hostility or aggression is frequently observed among these individuals [6]. Interestingly, hostile attribution biases are often reflected among individuals with alcohol dependence by their sensitivity to angry faces [7]. Thus, an investigation on how these individuals process angry faces compared to people that do not abuse alcohol may shed light onto important traits of alcohol abusers and may help target problem behaviors associated with a lack of anger management.

To date, we know that various brain regions are related to the perception of anger among healthy people, including the left ventrolateral orbitofrontal, right dorsolateral orbitofrontal, bilateral striate, and bilateral 
occipitotemporal regions [8]. Moreover, the emotional experience of anger is related to the left anterior insula, an affective division of the anterior cingulate cortex (ACC) [9], while rumination of anger is associated with the medial prefrontal cortex (MPFC) [10]. Salloum and colleagues [7] suggested that abnormalities in brain function, particularly dysfunction of the ACC, among individuals with alcohol dependence in evaluation of angry facial expressions. Salloum et al. determined this by presenting faces expressing relatively mild (30\%) and average (70\%) anger and instructed subjects to choose the intensity of each face. During this task, blood-oxygenation-level-dependent (BOLD) responses were measured, and it was determined that alcohol abusers presented greater activation in the ACC than non-alcohol abusers. However, this is the only study to investigate brain activation in relation to anger-associated stimuli among alcoholics. Moreover, previous studies lacked sufficient evidence to suggest any abnormality in a particular part of the brain with regard to processing anger.

In this study, we focused on the identification of specific brain regions associated with the emotional processing of anger among patients with alcohol dependence. Specifically, the goal was to explain how individuals with alcoholism process angry faces compared to healthy controls by observing BOLD brain responses during evaluation of angry facial expressions.

\section{Methods}

\section{Participants}

Eighteen male patients with alcohol dependency (Mean age: 49.83 years, age range $=39 \sim 60$ years) in an inpatient treatment facility voluntarily participated in this study. Specific inclusion criteria were as follows: 1) diagnosed as "Alcohol Dependent" by a psychiatrist based on DSM-IV criteria (American Psychiatric Association, 1994), 2) completion of detoxification, and 3) no history of concurrent psychiatric disorder(s). Although we initially recruited a total of 21 subjects, three individuals showed head motion artifacts in the functional magnetic resonance imaging (fMRI) scan and were hence excluded. With strict monitoring of the in-patient hospital, these individuals continued to stay sober from the time of their entry, with a minimum of 11 days to a maximum of 2,051 days of alcohol abstinence (median = $439.63, \mathrm{SD}=591.95)$. In consultation with their primary physician, patients on prescribed medication (that is, sleeping pills or anti-craving medication) were required to abstain from such medicines for 14 days prior to scanning.

Sixteen male non-alcoholic volunteers with similar demographics (mean age: 50.06 years, age range $=31 \sim 61$ years) were recruited as control subjects from the community via advertisements and flyers. Of the initially 17 recruited controls, one subject showed head motion artifacts during the fMRI and was hence excluded from our analyses. These volunteers did not report any history of impairment in the central nervous system or any psychiatric disease. The control subjects stayed abstinent from alcohol for at least $48 \mathrm{~h}$ prior to scanning.

The Korean version of the Alcohol Use Disorders Identification Test (AUDIT-K) [11] was administered to both groups to evaluate alcohol use; individuals that scored 15 points or higher met DSM-IV alcohol-use disorders criteria [12]. The Korean version of the Alcohol Dependence Scale (ADS-K) [13] was also administered to assess the level of alcohol dependency in patients suffering from alcoholism. The time frame of reporting any alcohol-related issues consisted of the 12 months prior to their hospitalization. Demographics and alcohol use of both patient and control groups are shown in Table 1.

\section{Facial stimuli}

We used five pictures of angry facial expressions excerpted from the Japanese Female Facial Expression database (JAFFE) [14]. Lyons et al. [14] conducted psychological experiments on 60 Japanese females using

Table 1 Demographics and alcohol use of study participants

\begin{tabular}{llll}
\hline Characteristics & Control group $(\boldsymbol{n}=16)$ & Patient group $(\boldsymbol{n}=\mathbf{1 8})$ & $\boldsymbol{t}$-value \\
\hline Age (years) & M(SD) & $49.83(6.60)$ & 0.11 \\
Educational level & $50.06(6.10)$ & $10.67(4.05)$ & 1.30 \\
Family history (\%) & $12.38(3.57)$ & 44.4 & $3.56^{* *}$ \\
Number of drinks (day per week) & 0 & $4.63(2.25)$ & $5.37^{* * *}$ \\
Amounts of drinks (drinks per drinking day) & $1.02(1.55)$ & $16.25(16.08)$ & $3.30^{* *}$ \\
Maximum number of drinks in a lifetime & $2.86(2.10)$ & $29.77(24.21)$ & $3.26^{* *}$ \\
AUDIT-K & $8.22(11.26)$ & $27.89(9.91)$ & $7.67^{* * *}$ \\
ADS-K & $6.38(5.54)$ & $50.00(12.85)$ & $6.34^{* * *}$ \\
\hline
\end{tabular}

${ }^{* * *} P<.001,{ }^{* *} P<.01$. Note. Means (standard deviations) are represented. One drink $=14 \mathrm{~g}$ ethanol. AUDIT-K Korean version of the Alcohol Use Disorders Identification Test, $A D S-K$ Korean version of the Alcohol Dependence Scale. 
JAFFE images where subjects were instructed to use a five-point Likert scale (" 1 " being "weak" to " 5 " being "intense") to rate the intensity of emotions displayed in each image. Pictures of facial expressions with an average rating of 4 points or higher on predominantly angry emotions were used for this study so that the facial expressions were intense enough for subjects to easily recognize anger and to also avoid any instance where subjects might confuse neutral emotions with anger. The person featured in each picture varied across all stimuli so as to parcel out any effect of familiarization. The average intensity of anger expressed in the facial stimuli was 4.51 points (SD 0.23).

\section{Procedures}

Subjects arrived in the laboratory $30 \mathrm{~min}$ prior to the experiment, received information about the experimental procedure, and signed the consent form. Afterwards, subjects completed the questionnaires on demographics and alcohol use, followed by a briefing on how to participate in the experiment.

The experimental task consisted of 10 blocks, that is, five fixation blocks and five emotional face blocks (that is, anger, fear, disgust, happiness, and sadness) lasting for $35 \mathrm{~s}$ per emotion. The fixation block preceded the emotional face block as a baseline, and a cross-hair ("+") was presented for the entire duration of the fixation block. During the emotional face block (that is, anger, fear, disgust, happiness, and sadness), five pictures of emotional facial expressions were presented for $7 \mathrm{~s}$ each, totaling $35 \mathrm{~s}$ per emotion. Subjects were asked to rate the intensity of each facial expression on a five-point Likert scale by pressing corresponding buttons specifically designed for this task. Consequently, the experimental task included five fixation blocks and five emotional face blocks, and lasted $350 \mathrm{~s}$ in total. Additionally, we counterbalanced the emotional face blocks to cancel out possible order effects across all participants. The experimental procedure during the entire period was conducted in strict compliance with the University Institutional Review Board.

\section{Imaging parameters}

Imaging was conducted on a $3.0 \mathrm{~T}$ whole-body ISOL Technology FORTE scanner (ISOL Technology, Korea) equipped with whole-body gradients and a quadrature head coil. Single-shot echo planar fMRI scans were acquired in 35 continuous slices parallel to the anterior commissure-posterior commissure line. Parameters for the fMRI included the following: repetition time/echo time (TR/TE) were $3,000 / 30 \mathrm{~ms}$, respectively, flip angle 80, field of view (FOV) $240 \mathrm{~mm}$, matrix size $64 \times 64$, slice thickness $4 \mathrm{~mm}$, and in-plane resolution $3.75 \mathrm{~mm}$. Three dummy scans from the beginning of the run were excluded to decrease the effect of non-steady-state longitudinal magnetization. T1-weighted anatomical images were obtained with a 3-D FLAIR sequence $(\mathrm{TR} / \mathrm{TE}=280 / 14 \mathrm{~ms}$, flip angle $=60, \mathrm{FOV}=240 \mathrm{~mm}$, matrix size $=256 \times 256$, slice thickness $=4 \mathrm{~mm}$ ).

\section{Data analysis}

Our main focus was on examining how patients with alcohol dependency responded to angry faces compared to normal controls; therefore, only results for the angry face condition were included in the data analyses. Using SPSS 20.0, an independent $t$-test was performed to compare the perceived level of anger intensity between the two groups. In the fMRI data analysis, brain scans from the fixation condition were compared to those obtained during the presentation of angry faces. The imaging data were analyzed with SPM8 (Wellcome Department of Cognitive Neurology, London, UK). All functional images were realigned with the image taken proximate to the anatomical study by using affine transformation routines built into SPM8. The realigned scans were normalized to SPM8' s template image that uses the space defined by the Montreal Neurologic Institute, which is very similar to the Talairach and Tournoux stereotaxic atlas [15]. Motion correction was done using sinc interpolation. The functional map was smoothed with a 8 -mm isotropic Gaussian kernel prior to statistical analysis. The voxel size was $2 \times 2 \times 2 \mathrm{~mm}^{3}$, resulting from normalization. In order to remove any artifacts resulting from cardio-respiration and other cyclical influences, time series data were filtered with a 240-s high-pass filter.

At the first level, the data were analyzed according to a standard box-car block design, after convolving the BOLD signal with a canonical HRF as modeled in SPM8. The individual first-level analyses of the comparisons of angry faces minus fixations were used for a random effect analysis and mean images for each subject were created. At the second level, mean images were combined in a one-sample $t$-test to assess significant group effects. In agreement with previous studies, we used a threshold of $P<0.001$ uncorrected, rather than the more rigorous $P<0.05$ corrected for the entire brain volume [16]. An extended threshold of 20 contiguous voxels was then applied to the activation. In the between-groups analysis, contrast images (angry face condition-fixation condition) were entered into a two-sample $t$-test (32 degrees of freedom). All coordinates derived from the statistical analysis were converted from MNI to the Talairach and Tournoux stereotaxic space [15].

To extract beta values from regions of interest (ROIs) (that is, ACC and MPFC), we selected activated clusters in each ROI using xjView (http://www.alivelearn.net/ xjview8/). In SPM, by creating a beta extraction batch file, we loaded the ROI image and assigned directories where beta value files for individual subjects were 
located. As the beta value could be calculated for each condition, we had beta values for $35 \mathrm{~s}$ of the angry face condition and $35 \mathrm{~s}$ of the fixation condition in both alcohol and control groups.

\section{Results}

\section{Behavioral results}

The mean (SD) of the intensity scores for control and patient groups were $3.72(0.53)$ and 3.34 (0.99), respectively. We found that controls and patients performed similarly in the rating of intensity for the expressions of anger $(t(32)=1.37$, NS).

\section{fMRI results}

Figure $1 \mathrm{~A}$ presents the brain regions that were activated in the control group during the angry face condition in reference to the fixation condition, while Figure $1 \mathrm{~B}$ is of the patient group. As for the control group, brain activation was observed in right dorsolateral prefrontal cortex (DLPFC) (Brodmann's Areas (BA) 46), bilateral culmen, left ventrolateral prefrontal cortex (VLPFC;BA 45)/left lateral orbitalfrontal cortex (LOFC; BA 47), right superior parietal lobule (BA 7), left inferior and middle temporal gyri (BA 20/39), right middle temporal gyrus (BA 21/37), left thalamus, and left superior temporal gyrus (BA 22)/left anterior temporal lobe (ATL; BA 38) (Figure 1A). A stark contrast was observed in the patient group results. That is, activated brain regions were the right superior frontal gyrus (BA 6), left inferior temporal gyrus (BA 20), left middle frontal gyrus (BA 6), left cingulate gyrus (BA 24), right postcentral gyrus (BA 43), right superior and middle temporal gyri (BA 21/ 38 ), left uncus/parahippocampal gyrus, and left precuneus (BA 7) (Figure 1B).
A

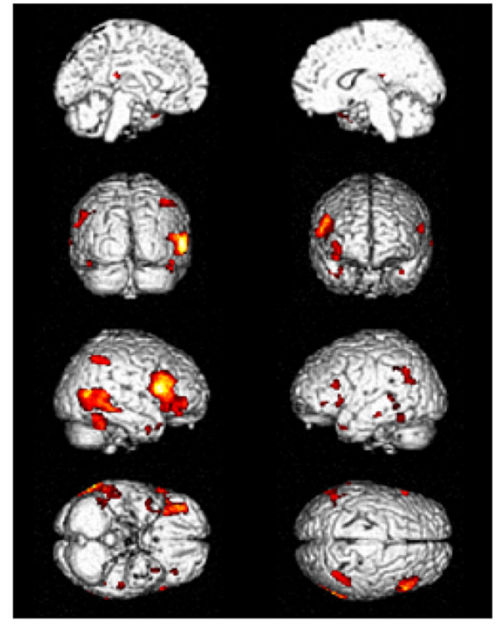

B

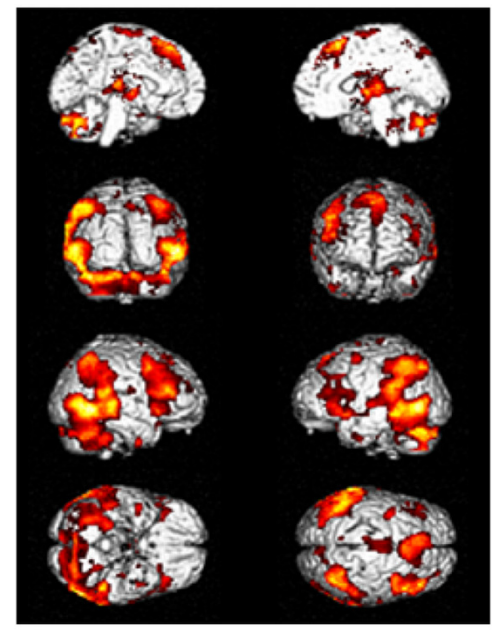

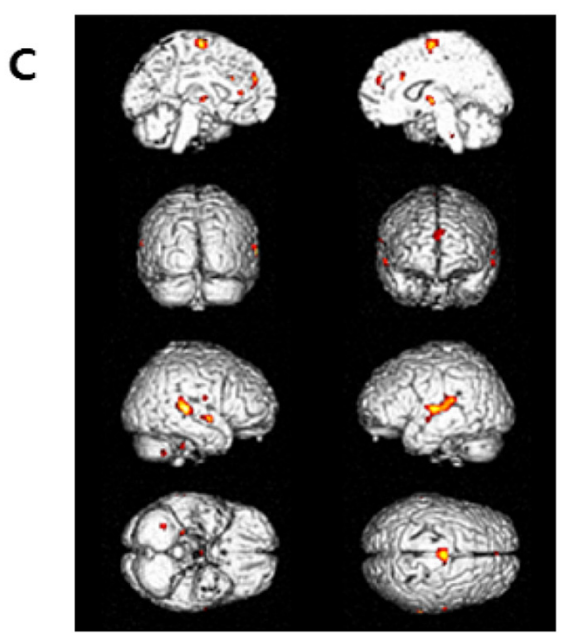

Figure 1 Brain regions activated during the angry face condition in reference to the fixation condition in each group, and the contrasting effects between the two groups (that is, the patient group versus control group). (A) the control group ( $n=16)$; (B) the patient group $(n=18)$; $(\mathbf{C})$ contrasting effects of brain activation in the patient group in reference to the control group (uncorrected $P<0.001$ ). 
Table 2 Talairach coordinates and $\boldsymbol{t}$-scores of activated brain areas

\begin{tabular}{|c|c|c|c|c|c|c|}
\hline Region & Side & $\mathrm{x}$ & $Y$ & $\mathbf{Z}$ & Brodmann's areas (BA) & $t$-value \\
\hline \multicolumn{7}{|l|}{ Angry face-fixation comparison } \\
\hline \multicolumn{7}{|l|}{ The control group } \\
\hline Dorsolateral prefrontal cortex & Right & 46 & 16 & 20 & 46 & 5.60 \\
\hline \multirow[t]{2}{*}{ Culmen } & Right & 46 & -46 & -28 & & 4.60 \\
\hline & Left & -44 & -52 & -18 & & 3.40 \\
\hline \multirow[t]{2}{*}{ Ventrolateral prefrontal cortex } & Left & -58 & 22 & 20 & 45 & 4.17 \\
\hline & Left & -44 & 16 & 0 & & 3.64 \\
\hline Lateral orbital frontal cortex & Left & -34 & 34 & -2 & 47 & 3.72 \\
\hline Superior parietal lobule & Right & 40 & -56 & 50 & 7 & 4.05 \\
\hline Inferior temporal gyrus & Left & -52 & -24 & -18 & 20 & 3.98 \\
\hline \multirow[t]{3}{*}{ Middle temporal gyrus } & Left & -58 & -66 & 24 & 39 & 3.66 \\
\hline & Right & 46 & 6 & -30 & 21 & 3.80 \\
\hline & Right & 64 & -52 & -2 & 37 & 5.15 \\
\hline Thalamus & Left & -6 & -30 & 16 & & 3.76 \\
\hline \multirow[t]{2}{*}{ Anterior temporal lobe } & Left & -34 & 10 & -32 & 38 & 3.64 \\
\hline & Left & -66 & -40 & 6 & 22 & 3.34 \\
\hline \multicolumn{7}{|l|}{ The patient group } \\
\hline Superior frontal gyrus & Right & 6 & 18 & 60 & 6 & 6.61 \\
\hline Inferior temporal gyrus & Left & -44 & -2 & -36 & 20 & 4.28 \\
\hline Middle frontal gyrus & Left & -34 & 0 & 54 & 6 & 4.26 \\
\hline Cingulate gyrus & Left & -20 & -12 & 44 & 24 & 4.18 \\
\hline Postcentral gyrus & Right & 66 & -10 & 20 & 43 & 4.04 \\
\hline Superior temporal gyrus & Right & 48 & 20 & -22 & 38 & 3.78 \\
\hline Middle temporal gyrus & Right & 50 & -30 & -8 & 21 & 8.18 \\
\hline Uncus/parahippocampal gyrus & Left & -24 & -2 & -26 & & 3.53 \\
\hline Precuneus & Left & -8 & -52 & 62 & 7 & 3.08 \\
\hline \multicolumn{7}{|l|}{ Contrasted brain activation areas } \\
\hline \multicolumn{7}{|c|}{ The patient group $>$ the control group } \\
\hline \multirow[t]{3}{*}{ Superior temporal gyrus } & Right & 62 & -32 & 8 & 22 & 4.70 \\
\hline & Right & 56 & -2 & -6 & 22 & 3.23 \\
\hline & Right & 40 & -30 & 8 & 41 & 3.20 \\
\hline Supplementary motor area & Right & 4 & -10 & 68 & 6 & 3.98 \\
\hline Superior temporal gyrus & Left & -56 & -16 & 6 & 41 & 3.68 \\
\hline Medial prefrontal cortex & Left & -2 & 50 & 30 & 9 & 3.40 \\
\hline Thalamus & Right & 6 & -10 & 2 & & 3.30 \\
\hline Postcentral gyrus & Right & 64 & -8 & 18 & 43 & 3.18 \\
\hline Dorsal anterior cingulate cortex & Left & -10 & 32 & 10 & 32 & 3.16 \\
\hline \multirow[t]{2}{*}{ Cerebellar tonsil } & Right & 32 & -56 & -44 & & 3.12 \\
\hline & Right & 24 & -32 & -38 & & 3.02 \\
\hline Cingulate gyrus & Right & 8 & 22 & 32 & 32 & 2.93 \\
\hline
\end{tabular}

Brain activation comparisons between the angry face and the fixation conditions in each group and contrasting effects between two groups (that is, the patient group versus control group) (uncorrected $P<0.001$ ). 
Measures of between-group differences provided clarification on the atypical anger-processing pattern seen in patients with alcohol dependency. As shown in Figure $1 \mathrm{C}$, when the brain activation observed between the angry face and fixation conditions of both groups were compared, the patient group exhibited significantly greater activity in the right supplementary motor area (SMA; BA 6), bilateral dorsal anterior cingulate cortex (dACC;BA 32), left medial prefrontal cortex (MPFC; BA 9), right thalamus, and bilateral superior temporal gyri. Talairach coordinates and $t$-scores of each activated area are shown in Table 2.

Extracted beta values for the $\mathrm{dACC}$ and MPFC regions in both groups for each experimental condition (that is, angry face and fixation conditions) are shown in Figure 2A, B. When we conducted small-volume corrections on the ROIs (that is, $\mathrm{AACC}$ and MPFC), we were not able to find significant voxels. However, results from an independent $t$-test (SPSS 20.0) with the ROI beta values (the angry face condition - the fixation condition) showed a significant difference between control and alcohol-dependent groups (dACC: $t(32)=$ 3.167, $P<0.01$; MPFC: $t(32)=3.316, P<0.01)$.

\section{Discussion}

The findings of the current study are as follows. In the control group, brain activation was observed during the processing of angry faces in the right DLPFC (BA 46), left VLPFC (BA 45), left LOFC (BA 47), and left ATL (BA 38). This is consistent with what has been observed of the brain regions involved in anger perception or experience [8]. The OFC, in particular, has been shown as a brain area activated when encountered with angry facial expressions among normal people [17], and the results of our control group echo this finding.

Brain activation during anger processing in patients with alcoholism differed from the control group in a number of cortical regions. A finding that draws particular interest is the significantly greater activation that was observed in the bilateral dACC (BA 32) among the patient group during the anger condition. The ACC is involved in attention but is also activated during heightened anger [10]. Interestingly, this region seems to be more involved in anger control than in the actual expression of anger per se [18]. For instance, recent reviews of the ACC by Shenhav et al. [19] and Gasquoine [20] note that the "ACC contributes to behavior by modifying responses especially in reaction to challenging cognitive and physical states that require additional effortful cognitive control." This idea is similar to Eisenberger and Lieberman's notion of the ACC as a "neural alarm system" [21]. Thus, in relation to the current study, ACC activation may be indicative of inefficient functioning and/or hypersensitivity to angry
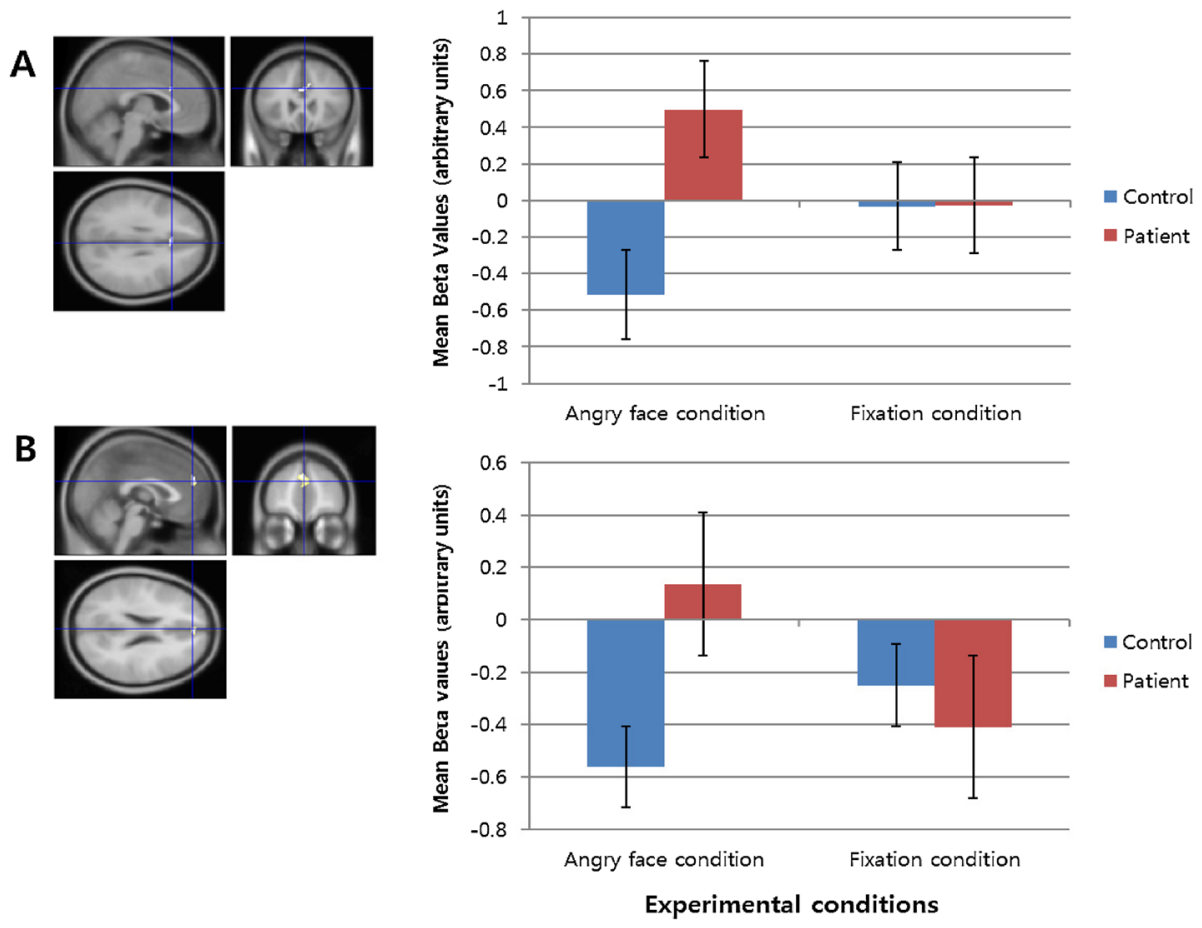

Figure 2 The extracted beta values for the bilateral dACC (A) and left MPFC (B) (average beta value \pm SD) in the control and patient groups for each experimental condition (that is, angry face and fixation conditions). 
faces in participants that were alcohol-dependent. In other words, participants with alcohol dependency require recruitment of a wider network of regions implicated in cognitive control and self-regulation than healthy controls.

We also found that, during evaluation of angry facial expressions, participants that were alcohol-dependent showed greater activation in the MPFC (BA 9). The greater MPFC activation in patients with alcohol dependency could indicate more rumination [10]. However, because the presentation of angry faces and the subsequent rating of their intensity may not completely elicit rumination, it is plausible to suggest that the MPFC activation may reflect inefficient or hypersensitive social cognitive processing when individuals encounter threatening angry faces. This is because along with its involvement in angry rumination, the MPFC is more broadly associated with social cognition [22].

Despite our findings showing an abnormality in brain functioning when patients with alcoholism are faced with intense anger, they did not differ from the control group in terms of behavioral outcomes on perceived anger intensity. This may be due to the possibility that our stimuli and procedure could not precisely detect the behavioral malfunction of patients with alcohol dependency when processing anger. An alternative explanation could be an issue with the intensity of the stimuli. More specifically, a ceiling effect may have occurred that made it evidently easy for subjects to identify anger, yet not sensitive enough to detect varying degrees of behavioral issues. On the other hand, it is conceivable that individuals with alcohol abuse may have experienced difficulty in self-reporting their perceived level of emotion. In fact, this has been supported by previous studies showing that individuals with alcoholism, despite lower performance scores, report equivalent degrees of difficulty in completing emotional-decoding tasks as did normal controls [23]. Given this issue, the patient group might not have accurately reported their perceived level of anger.

This study has a number of limitations. First, the period of alcohol abstinence varied across study participants (from 11 to 2,051 days), which made it difficult to suggest a relationship between the length of abstinence and their anger perception. Second, our study did not control for possible sleep-deprivation issue among patients that were alcohol-dependent. This may be an arguable issue given that frequent awakening after sleep onset is often seen in patients with alcohol dependency [24]. Moreover, insomnia and/or sleep deprivation have been linked with inaccurate recognition of human emotions, thus, limited ability to control for anger ventilation [25].

Various clinical studies have emphasized hypersensitivity to threats among individuals with alcohol dependence and that these individuals experience intense emotions to negative stimuli. In that sense, despite their limitations, our findings are unique in that greater activation in the $\mathrm{dACC}$ and MPFC might suggest inefficient functioning and/or hypersensitivity when patients with alcohol dependency process angry faces.

\section{Competing interests}

The authors declare that they have no competing interests.

\section{Authors' contributions}

MSP, GJK, and JHS have made substantial contributions to conception and design, or acquisition of data, or analysis and interpretation of data. SHK has been involved in drafting the manuscript or revising it critically for important intellectual content. SJS and YKK agree to be accountable for all aspects of the work in ensuring that questions related to the accuracy or integrity of any part of the work are appropriately investigated and resolved. All authors read and approved the final manuscript.

\section{Acknowledgements}

This research paper is made possible through the help and support from Ms. Jihye Noh and Ms. Ji-Eun Park. I would like to thank Ms. Jihye Noh for her most support in order to conduct the fMRI experiment with alcoholic patients. Ms. Ji-Eun Park kindly read my paper and offered invaluable detailed advices on grammar, organization, and the theme of the paper. I would like to also thank to my participants who made this study possible. This work was supported by the National Research Foundation of Korea Grant funded by the Korean Government (No. NRF-2006-2005087).

\section{Author details}

'Department of Psychology, Chungnam National University, 99 Daehak-ro, Yuseong-gu, Daejeon 305-764, South Korea. ${ }^{2}$ Graduate School of Health and Complementary Medicine, Wonkwang University, 460 Iksandae-ro, Iksan, Jeonbuk 570-749, South Korea. ${ }^{3}$ Department of Social Welfare, Cheongju University, 298 Daeseong-ro, Cheongju-si, Chungcheongbuk-do 363-764, South Korea. ${ }^{4}$ Department of Psychiatry, Hanmaum Alcohol Treatment Center, 513-1 Jangan-dong, Seo-gu, Daejeon, South Korea. ${ }^{5}$ Department of Industrial Design, Graduate school of Integrated Frontier Science, Faculty of Design, Kyushu University, 4-9-1 Shiobaru, Minami-ku, Fukuoka 815-8540, Japan.

Received: 24 September 2014 Accepted: 4 February 2015

Published online: 01 March 2015

\section{References}

1. Schonwetter DJ, Janisse MP. Alcohol consumption, anger and hostility: a link to coronary heart disease. Pers Indiv Differ. 1991;12:1049-55.

2. Tivis LJ, Parsons OA, Nixon SJ. Anger in an inpatient treatment sample of chronic alcoholics. Alcohol Clin Exp Res. 1998;22:902-7.

3. McNair DM, Lorr M, Droppelman LF. Manual for the profile of mood states. Educational and Industrial Testing Services: San Diego; 1971.

4. Parrott DJ, Zeichner A, Stephens D. Effects of alcohol, personality, and provocation on the expression of anger in men: a facial coding analysis. Alcohol Clin Exp Res. 2003;27:937-45.

5. Kelly JF, Magill M, Stout RL. How do people recover from alcohol dependence? A systematic review of the research on mechanisms of behavior change in Alcoholics Anonymous. Addict Res Theory. 2009;17:236-59.

6. Handelsman L, Stein JA, Bernstein DP, Oppenheim SE, Rosenblum A, Magura S. A latent variable analysis of coexisting emotional deficits in substance abusers: alexithymia, hostility, and PTSD. Addict Behav. 2000;25:423-8

7. Salloum JB, Ramchandani VA, Bodurka J, Rawlings R, Momenan R, George D, et al. Blunted rostral anterior cingulate response during a simplified decoding task of negative emotional facial expressions in alcoholic patients. Alcohol Clin Exp Res. 2007:31:1490-504.

8. Lindquist KA, Wager TD, Kober H, Bliss-Moreau E, Barrett LF. The brain basis of emotion: a meta-analytic review. Behav Brain Sci. 2012;35:121-43.

9. Dougherty DD, Shin LM, Alpert NM, Pitman RK, Orr SP, Lasko M, et al. Anger in healthy men: a PET study using script-driven imagery. Biol Psychiatry. 1999;46:466-72. 
10. Denson TF, Pedersen WC, Ronquillo J, Nandy AS. The angry brain: neural correlates of anger, angry rumination, and aggressive personality. J Cogn Neurosci. 2009;21:734-44.

11. Kim JS. A understanding about recovery process of alcoholism. J Korean Acad Fam Med. 1998;19:304-12.

12. Park BK, Kim JS, Lee DB. Comparison of four alcoholism screening tools based on sensitivity and specificity for DSM-IV criteria. J Korean Acad Fam Med. 2000;21:1427-35.

13. Lee DK, Shin JK, Yun SM, Byun WT. A reliability and validity study of the Korean Version of the Alcohol Dependence Scale in alcoholics. J Korean Acad Addict Psychiatry. 2000;4:30-7.

14. Lyons MJ, Akamatsu S, Kamachi M, Gyoba J. Coding facial expressions with Gabor Wavelets. Third IEEE Proceedings of International Conference on Automatic Face and Gesture Recognition. Nara Japan: IEEE Computer Society; 1998.

15. Talairach J, Tournoux P. Co-planar stereotaxic atlas of the human brain. New York: George Thieme Verlag; 1988.

16. Friston KJ, Holmes AP, Price CJ, Buchel C, Worsley KJ. Multisubject fMRI studies and conjunction analyses. Neuroimage. 1999;10:385-96.

17. Blair RJR, Morris JS, Frith CD, Perrett DI, Dolan RJ. Dissociable neural responses to facial expressions of sadness and anger. Brain. 1999;122:883-93.

18. Denson TF, Dobson-Stone C, Ronay R, von Hippel W, Schira MM. A functional polymorphism of the MAOA gene is associated with neural responses to induced anger control. J Cogn Neurosci. 2014;26:1418-27.

19. Shenhav A, Botvinick MM, Cohen JD. The expected value of control: an integrative theory of anterior cingulate cortex function. Neuron. 2013;79:217-40.

20. Gasquoine PG. Localization of function in anterior cingulate cortex: from psychosurgery to functional neuroimaging. Neurosci and Biobehav R. 2013;37:340-8.

21. Eisenberger $\mathrm{NI}$, Lieberman MD. Why rejection hurts: a common neural alarm system for physical and social pain. Trends Cogn Sci. 2004;8:294-30.

22. Ochsner KN. The social-emotional processing stream: five core constructs and their translational potential for schizophrenia and beyond. Biol Psychiatry. 2008;64:48-61.

23. Philippot P, Kornreich C, Blairy S, Baert I, Den Dulk A, Le Bon O, et al Alcoholics' deficits in the decoding of emotional facial expression. Alcohol Clin Exp Res. 1999;23:1031-8.

24. Brower KJ. Insomnia, alcoholism and relapse. Sleep Med Rev. 2003;7:523-39.

25. van der Helm E, Gujar N, Walker MP. Sleep deprivation impairs the accurate recognition of human emotions. Sleep. 2010;33:335-42

\section{Submit your next manuscript to BioMed Central and take full advantage of:}

- Convenient online submission

- Thorough peer review

- No space constraints or color figure charges

- Immediate publication on acceptance

- Inclusion in PubMed, CAS, Scopus and Google Scholar

- Research which is freely available for redistribution 